\title{
Nedaplatin- versus cisplatin-based chemotherapy in the survival time of patients with non-small cell lung cancer
}

\author{
JINLU SHAN*, YANLI XIONG ${ }^{*}$, DONG WANG, MINGFANG XU, YI YANG, \\ KAN GONG, ZHENZHOU YANG, GE WANG and XUEQIN YANG
}

\author{
Cancer Center, Daping Hospital, Third Military Medical University, Chongqing 400042, P.R. China
}

Received September 28, 2014; Accepted January 28, 2015

DOI: $10.3892 / \operatorname{mco} .2015 .504$

\begin{abstract}
Nedaplatin (NDP) has been extensively used to treat patients with non-small cell lung cancer (NSCLC) in the last decade. The present study compared the survival benefits of NDP and cisplatin (DDP) in the treatment of NSCLC. Patients $(n=392)$ with NSCLC were treated with at least two cycles of platinum-based chemotherapy. Among these patients, 202 received DDP-based chemotherapy, and 190 received NDP-based chemotherapy. The overall survival time of the two groups and the toxicity of drugs were analyzed. The results showed that only the chemotherapy cycle duration was found to be statistically different between DDP and NDP groups in all the characteristics. The mean chemotherapy duration was 3.3 cycles in the DDP group, and 4.1 cycles in the NDP group $\left(\chi^{2}=20.206, \mathrm{P}<0.001\right)$. Additionally, the chemotherapy cycle number was also an independent predictive factor for the overall survival time in the multivariate analysis $(\mathrm{HR}=0.539$, $\mathrm{P}<0.001)$. The median survival time (MST) was 15 months in the DDP group, and 20 months in the NDP group $\left(\chi^{2}=5.189\right.$, $\mathrm{P}=0.023)$. The 1-, 2- and 3-year overall survival rates were $62.4,25.7$ and $15.8 \%$, and $78.9,38.9$, and $16.8 \%$ in the DPP and NDP groups, respectively. The incidence of grade 3-4 nausea/vomiting, anorexia and weight loss was higher in the DDP compared to the NDP group (36.1 vs. 8.4\%, 17.3 vs. $5.8 \%$, and 9.9 vs. $1 \%$, respectively). In conclusion, NDP-based chemotherapy had a survival benefit compared to DDP-based chemotherapy for NSCLC patients, due to the lower toxicity of NDP, which renders this drug more tolerable, thus allowing patients to undergo more cycles of chemotherapy.
\end{abstract}

Correspondence to: Dr Xueqin Yang, Cancer Center, Daping Hospital, Third Military Medical University, 10 Changjiang Zhilu, Daping Yuzhong, Chongqing 400042, P.R. China

E-mail: yangxueqin@hotmail.com

*Contributed equally

Key words: nedaplatin, chemotherapy, survival, non-small cell lung cancer

\section{Introduction}

Lung cancer is a leading cause of cancer-related mortality worldwide and is expected to remain a major health problem (1). The morbidity and mortality rates of lung cancer in China are the highest among all the malignant tumors. The majority of patients are diagnosed at an advanced stage, at which the cancer is inoperable. Thus, chemotherapy has become the primary treatment. However, the adverse effects of certain agents, which lead to failure to complete the scheduled regimen, extension of chemotherapy intervals or reduction of the recommended dosage, have limited their clinical application. Therefore, it is extremely important to investigate and identify effective chemotherapy agents with low toxicity.

Nedaplatin [NDP; cis-diamine (glycolate) platinum II] is a second-generation platinum analog, synthesized by Shionogi \& Co. Ltd. (Osaka, Japan). NDP has a higher aqueous solubility than cisplatin (DDP), and was found to be highly effective against solid tumors, in preclinical studies (2-4). Koshiyama et al (5) reported that the mean tumor inhibition rate for NDP was equal to or higher than that for DDP in 15 cervical (70.7 vs. $63.9 \%), 65$ ovarian (61.7 vs. $54.8 \%)$ and 57 endometrial (52.1 vs. $47.7 \%$ ) carcinoma patients. Compared to DDP, NDP-induced emesis and nephrotoxicity are substantially reduced, bypassing the requirement for hydration therapy for renal protection (6). The dose-limiting toxicity of NDP is characterized by thrombocytopenia.

Numerous cancers, including nasopharyngeal cancer, NSCLC, esophageal cancer, urothelial carcinoma and cervical cancer, have been reported to be effective to NDP-based chemotherapy in clinical studies (7-13). However, the majority of recent studies have focused on the therapeutic effect of NDP on esophageal cancer, although this type of cancer does not respond well to platinum-based chemotherapy. Limited studies have addressed the effect of NDP on the treatment of lung cancer. Sasaki et al (14) reported that NDP shows equivalent antitumor activity to DDP against lung cancer cell lines in vitro. Furuse et al (15) reported that a combination of NDP and vindesin (VDS) was a safe and effective regimen for the treatment of NSCLC, generating antitumor effects equivalent to that of the DDP/VDS regimen. Thus far, no study has compared the survival benefit between NDP and DDP in the treatment of NSCLC. 
In the last decade, NDP-based chemotherapy has been extensively used in Chinese NSCLC patients (16). The present study reports a retrospective study comparing the efficacy of NDP and DDP in the treatment of NSCLC. In the study, a retrospective analysis based on 392 patients diagnosed with NSCLC revealed that NDP-based chemotherapy increased the median survival time (MST) of NSCLC patient compared to DDP. The observed survival benefit is due to the reduced toxicity of NDP, which allows patients to tolerate more cycles of chemotherapy.

\section{Patients and methods}

Eligibility criteria. A total of 966 patients diagnosed with NSCLC at the Cancer Center of Daping Hospital at the Third Medical University (Chongqing, China), in the period between January 2003 and December 2007 were retrospectively reviewed. Every patient was evaluated for age, gender, smoking status, stage, histology type, chemotherapy regimen, overall chemotherapy cycles and other treatments. Eligibility criteria for the study were as follows: Histological or cytological confirmation of NSCLC, previously untreated with chemotherapy, at least two cycles of platinum-based therapy (DDP- or NDP-based chemotherapy), no surgical treatment of the primary site and no changing to a different platinum agent or to a non-platinum regimen in a subsequent treatment. Based on the above criteria, a total of 392 NSCLC patients were selected. Among them, 202 patients received DDP-based chemotherapy and 190 patients received NDP-based chemotherapy. Table I shows that the two patient groups were not significantly different in terms of demographics, disease severity and treatment regimen.

Clinical data from these patients were acquired and stored according to protocols approved by the local ethics committee.

Treatment schedule. The patients received one of the following combination chemotherapies by intravenous injection: Gemcitabine + platinum (GP), paclitaxel + platinum (TP), navelbine + platinum (NP), docetaxel + platinum (DP) and cyclophosphamide + doxorubicin + platinum $(\mathrm{CAP})$. In each regimen, the platinum-based compound was either DDP or NDP. The dose of gemcitabine was $1000 \mathrm{mg} / \mathrm{m}^{2}$ on days 1 and 8 ; docetaxel was $75 \mathrm{mg} / \mathrm{m}^{2}$ on day 1 ; paclitaxel was $135-175 \mathrm{mg} / \mathrm{m}^{2}$ on day 1 ; navelbine was $25 \mathrm{mg} / \mathrm{m}^{2}$ on days 1 and 8; cyclophosphamide was $600 \mathrm{mg} / \mathrm{m}^{2}$ on day 1 ; doxorubicin was $50 \mathrm{mg} / \mathrm{m}^{2}$ on day 1 ; and DDP and NDP were $80 \mathrm{mg} / \mathrm{m}^{2}$ on day 1 .

All the patients received dexamethasone and the 5-hydroxytryptamine receptor antagonist on days 1,2 and 3, or days 8 and 9 , to prevent chemotherapy-induced nausea and vomiting. Dexamethasone was also used prior to the administration of paclitaxel, to prevent allergic reaction. Hydration with 3 to 61 of intravenous fluids and mannitol was conducted before and at the day of the administration of DDP. All the chemotherapy regimens were repeated every 21-28 days. Chemotherapy was continued until unacceptable toxicity was observed, or until the patient refused further treatment.

Evaluation. The overall survival time and toxicities observed were analyzed in each patient group. Overall survival time
Table I. Patient characteristics.

\begin{tabular}{|c|c|c|c|c|}
\hline Variables & $\begin{array}{c}\mathrm{DDP}, \mathrm{n}(\%) \\
(\mathrm{n}=202)\end{array}$ & $\begin{array}{c}\text { NDP, n (\%) } \\
\quad(\mathrm{n}=190)\end{array}$ & $\chi^{2}$ & P-value \\
\hline \multicolumn{5}{|l|}{ Gender } \\
\hline Female & $61(30.2)$ & $50(26.3)$ & 0.727 & 0.394 \\
\hline Male & $141(69.8)$ & $140(73.7)$ & & \\
\hline \multicolumn{5}{|l|}{ Age, years } \\
\hline$<60$ & $110(54.5)$ & $103(54.2)$ & 0.002 & 0.961 \\
\hline$\geq 60$ & $92(45.5)$ & $87(45.8)$ & & \\
\hline \multicolumn{5}{|l|}{ Smoking status } \\
\hline Non-smoker & $105(52.0)$ & $89(46.8)$ & 1.034 & 0.309 \\
\hline Current smoker & $97(48.0)$ & $101(53.2)$ & & \\
\hline \multicolumn{5}{|l|}{ Histology type } \\
\hline $\mathrm{Sq}$ & $74(37.6)$ & $58(30.5)$ & 2.192 & 0.139 \\
\hline Non-Sq & $128(62.4)$ & $132(69.5)$ & & \\
\hline \multicolumn{5}{|l|}{ Stage } \\
\hline I-II & $18(8.9)$ & $22(11.6)$ & 4.371 & 0.112 \\
\hline III & $118(58.4)$ & $91(47.9)$ & & \\
\hline IV & $66(32.7)$ & $77(40.5)$ & & \\
\hline \multicolumn{5}{|l|}{ Regimen } \\
\hline GP & $24(11.9)$ & $30(15.8)$ & 39.706 & $<0.001$ \\
\hline TP & $114(56.4)$ & $90(47.4)$ & & \\
\hline DP & $24(11.9)$ & $61(32.1)$ & & \\
\hline CAP & $22(10.9)$ & $3(1.6)$ & & \\
\hline NP & $18(8.9)$ & $6(3.2)$ & & \\
\hline \multicolumn{5}{|l|}{ Cycles } \\
\hline $2-3$ & $114(56.4)$ & $70(36.8)$ & 20.206 & $<0.001$ \\
\hline $4-5$ & $70(34.7)$ & $78(41.1)$ & & \\
\hline$\geq 6$ & $18(8.9)$ & $42(22.1)$ & & \\
\hline \multicolumn{5}{|l|}{ Other treatments } \\
\hline Radiotherapy & $162(80.2)$ & $137(72.1)$ & 1.796 & 0.180 \\
\hline Target therapy & $11(5.4)$ & $16(8.4)$ & & \\
\hline
\end{tabular}

Sq, squamous cancer; GP, gemcitabine + platinum; TP, paclitaxel + platinum; DP, docetaxel + platinum; CAP, cyclophosphamide + doxorubicin+platinum; NP, navelbine + platinum.

was calculated from the first day of chemotherapy until the last follow-up or until the patient succumbed. For patients with longer survival times, follow-up was discontinued at 5 years. The severity of all the toxicities associated with chemotherapy was assessed according to the National Cancer Institute Common Terminology Criteria for Adverse Events (version 3.0) grading system (17). Anemia, neutropenia, thrombocytopenia, nausea/vomiting, anorexia, renal toxicity, neurotoxicity and weight loss were the symptoms of toxicities that were evaluated.

Statistical analysis. Statistical analysis was performed using a statistical software package (SPSS for Windows, version 13.0; SPSS, Chicago, IL, USA). Survival curves were estimated using the Kaplan-Meier method, with censoring to correct for loss to follow-up. Survival difference was analyzed by 
the log-rank test. Multivariate analysis was performed with the Cox proportional hazards model. To calculate statistical significance between categorical variables, $\chi^{2}$ or the Fisher exact test were used. Pearson correlation analysis was used to assess the association between the two groups. Two-tailed $\mathrm{P}$-values were assessed and $\mathrm{P}<0.05$ was considered to indicate a statistically significant difference.

\section{Results}

Patient characteristics. The retrospective study analyzed a total number of 392 patients diagnosed with NSCLC between January 2003 and December 2007. Among them, 202 had received DDP-based chemotherapy and 190 had received NDP-based chemotherapy. The patient characteristics are shown in Table I. No statistical difference was observed between the two groups with regard to gender, age, smoking status, histology type and stage, as analyzed by the Pearson $\chi^{2}$ test $(\mathrm{P}<0.05)$.

All the enrolled patients had received at least two cycles of chemotherapy. The mean chemotherapy duration was 3.3 cycles in the DDP and 4.1 cycles in the NDP group. The number of patients receiving $>4$ chemotherapy cycles was $88 / 202$ (43.6\%) for the DDP group and 120/190 (63.2\%) for the NDP group $\left(\chi^{2}=20.206, \mathrm{P}<0.001\right.$, Table I).

Enrolled patients had undergone multiple regimens as the first-line regimen, including GP, TP, DP, NP and CAP. Although there were significant differences between the DDP and NDP groups $\left(\chi^{2}=39.706, \mathrm{P}<0.001\right), \sim 50 \%$ of patients had received the TP regimen in each group (Table I).

Certain patients received radiotherapy or targeted therapy in the subsequent treatment, but there was no statistical difference between the percentages of patients receiving this therapy in the two groups (Table I).

Survival. Overall survival (OS) was considered from the start of treatment to the date of data analysis or the date of loss from follow-up for the remaining patients. The median follow-up time was 28 months (range, 4-60 months). As a result, the MST was 15 months [95\% confidence interval (CI), 13.4-16.6] for the DDP group and 20 months (95\% CI, 17.0-23.0) for the NDP group. Statistical analysis indicated that the NSCLC patients treated with NDP survived significantly longer than those with DDP $\left(\chi^{2}=5.189, P=0.023\right)$ (Table II and Fig. 1). Multivariate analyses showed that the type of platinum agent used was an independent predictive factor for the overall survival time of NSCLC patients [hazard ratio (HR), 0.764; 95\% CI, 0.606-0.963; P=0.022] (Table II). The 1-, 2- and 3-year overall survival rates were $62.4,25.7$ and $15.8 \%$ for the DDP group, and 78.9, 38.9 and $16.8 \%$ for the NDP group, respectively. A statistical difference was observed between the two groups in the 1 - and 2-year overall survival rates $\left(\chi^{2}=13.904\right.$, $\mathrm{P}<0.001 ; \chi^{2}=7.827, \mathrm{P}=0.005$, respectively).

From Table II, the chemotherapy cycle number was an independent predictive factor for the overall survival time of NSCLC patients (HR, 0.539; 95\% CI, 0.451-0.643; $\mathrm{P}<0.001$ ). Table III showed that the MST of the patients with 2-3, 4-5 and $\geq 6$ chemotherapy cycles in the DDP group and in the NDP group was 10, 18 and 24 months vs. 12, 20 and 26 months, respectively. However, no statistical difference was identified

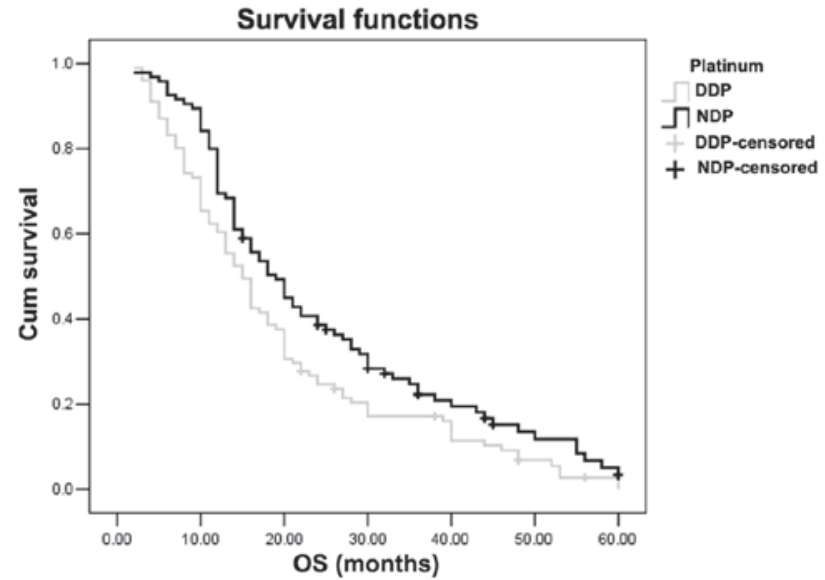

Figure 1. Kaplan-Meier analysis of overall survival (OS) times in patients receiving different platinum-based treatments. MST was 15 months (95\% CI, 3.4-16.6) in the DDP group and 20 months (95\% CI: 17-23) in the NDP group (Log-rank test: $\left.\chi^{2}=5.189, \mathrm{P}=0.023\right)$. MST, median survival time; $\mathrm{CI}$, confidence interval; DDP, cisplatin; NDP, nedaplatin.

between the DDP and NDP groups $\left(\chi^{2}=0.040, \mathrm{P}=0.980\right)$. Table III also showed that NDP-based chemotherapy was beneficial regardless of smoking status. No statistical differences were observed between the two groups for female patients, patients aged $>60$ years and patients with non-squamous cancer. However, younger patients ( $<60$ years), male patients, patients with squamous cancer and stage III in the NDP group had a longer survival time compared to patients with the same characteristics in the DDP group (21 vs. 16 months, $\mathrm{P}<0.001$; 20 vs. 14 months, $\mathrm{P}<0.001 ; 24$ vs. 16 months, $\mathrm{P}=0.021$; and 20 vs. 15 months, $\mathrm{P}<0.001$, respectively). For further study in these subgroups, the distribution of the chemotherapy cycles was significantly different in the different platinum agent groups (Fig. 2). Thus, the chemotherapy cycles were the main reason that caused the different survival time.

Table II also showed that stage was an independent predictive factor for the overall survival time of NSCLC patients $(\mathrm{HR}=2.099 ; 95 \% \mathrm{CI}, 1.756-2.510 ; \mathrm{P}<0.001)$. As the characteristic baseline regarding stage between DDP and NDP groups was balanced, further analysis was not performed.

Toxicity. The hematological and non-hematological toxicities are summarized in Table IV. No grade 3 or 4 renal toxicity or neurotoxicity was observed in either of the two groups. A significant difference was observed in thrombocytopenia, nausea/vomiting, anorexia and weight loss between the two groups. The rates of thrombocytopenia were higher in the NDP compared to the DDP group (12.1 vs. 5.4\%, $\mathrm{P}=0.019$ ). However, the rates of nausea/vomiting, anorexia and weight loss were higher in the DDP compared to the NDP group (36.1 vs. $8.4 \%, \mathrm{P}<0.001 ; 17.3$ vs. $5.8 \%, \mathrm{P}<0.001$; and 9.9 vs. $1.0 \%, \mathrm{P}<0.001$, respectively).

\section{Discussion}

Chemotherapy is the major method for treatment of lung cancer, owing to its high mortality and morbidity rates; recently, the use of platinum-based chemotherapeutic agents have allowed for significant advances in the survival of patients 
Table II. Association between patient characteristics and overall survival time.

\begin{tabular}{|c|c|c|c|c|c|}
\hline Variables & $\mathrm{n}$ & $\begin{array}{l}\text { MST, months } \\
\quad(95 \% \mathrm{CI})\end{array}$ & $\begin{array}{c}\text { P-value } \\
\text { (Univariate analysis) }\end{array}$ & $\begin{array}{c}\mathrm{HR} \\
(95 \% \mathrm{CI})\end{array}$ & $\begin{array}{c}\text { P-value } \\
\text { (Multivariate analysis) }\end{array}$ \\
\hline \multicolumn{6}{|l|}{ Gender } \\
\hline Female & 111 & $16(13.1-18.9)$ & 0.380 & $0.952(0.694-1.307)$ & 0.761 \\
\hline Male & 281 & $17(13.1-18.9)$ & & & \\
\hline \multicolumn{6}{|l|}{ Age, years } \\
\hline$<60$ & 212 & $18(16.2-19.8)$ & 0.066 & $0.994(0.791-1.249)$ & 0.958 \\
\hline$\geq 60$ & 180 & $15(12.7-17.3)$ & & & \\
\hline \multicolumn{6}{|l|}{ Smoking status } \\
\hline Non-smoker & 194 & $18(15.6-20.4)$ & 0.241 & $0.829(0.616-1.115)$ & 0.214 \\
\hline Current smoker & 198 & $16(13.9-18.1)$ & & & \\
\hline \multicolumn{6}{|l|}{ Histology type } \\
\hline $\mathrm{Sq}$ & 132 & $18(15.7-20.3)$ & 0.102 & $0.898(0.682-1.164)$ & 0.416 \\
\hline Non-Sq & 260 & $16(14.0-18.0)$ & & & \\
\hline \multicolumn{6}{|l|}{ Stage } \\
\hline I-II & 40 & $36(29.5-50.5)$ & $<0.001$ & $2.099(1.756-2.510)$ & $<0.001$ \\
\hline III & 209 & $17(14.8-19.2)$ & & & \\
\hline IV & 143 & $13(11.8-14.2)$ & & & \\
\hline \multicolumn{6}{|l|}{ Regimen } \\
\hline GP & 54 & $18(16.8 .0-23.2)$ & 0.060 & $0.952(0.866-1.046)$ & 0.304 \\
\hline $\mathrm{TP}$ & 204 & $15(12.6-17.4)$ & & & \\
\hline DP & 85 & $17(15.4-18.6)$ & & & \\
\hline CAP & 25 & $14(9.4-18.6)$ & & & \\
\hline NP & 24 & $18(12.8-23.2)$ & & $0.539(0.451-0.643)$ & $<0.001$ \\
\hline \multicolumn{6}{|l|}{ Cycles } \\
\hline $2-3$ & 184 & $12(10.6-13.4)$ & $<0.001$ & & \\
\hline $4-5$ & 148 & $19(17.7-24.3)$ & & & \\
\hline$\geq 6$ & 60 & $23(19.8-32.2)$ & & & \\
\hline \multicolumn{6}{|l|}{ Platinum } \\
\hline DDP & 202 & $15(13.4-16.6)$ & 0.023 & $0.764(0.607-0.962)$ & 0.022 \\
\hline NDP & 190 & $20(17.0-2.30)$ & & & \\
\hline
\end{tabular}

Sq, squamous cancer; GP, gemcitabine + platinum; TP, paclitaxel + platinum; DP, docetaxe + platinum; CAP, cyclophosphamide + doxorubicin + platinum; NP, navelbine + platinum; DDP, cisplatin; NDP, nedaplatin; MST, median survival time; HR, hazard ratio.
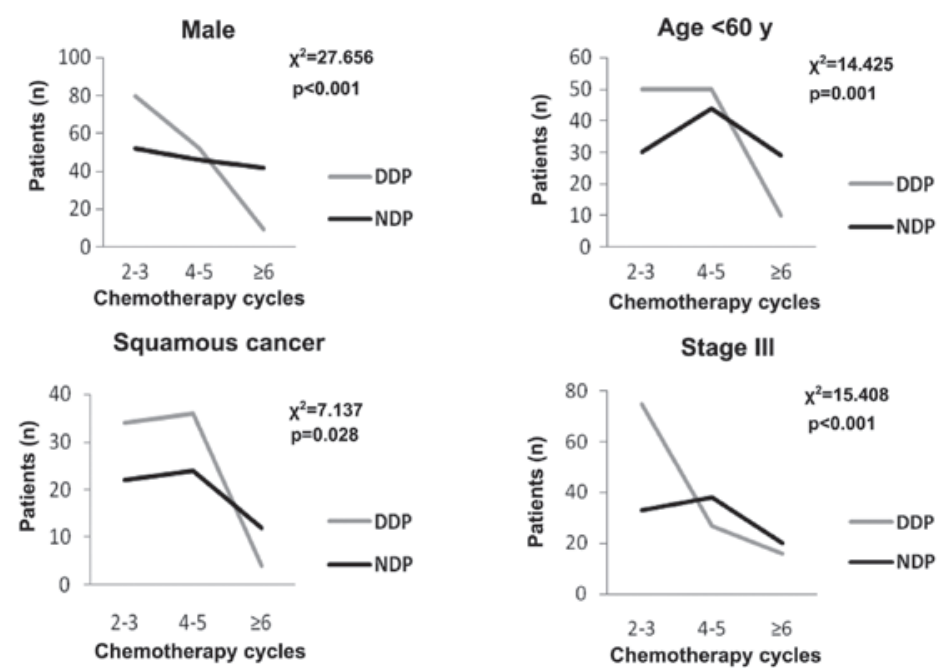

Figure 2. $\chi^{2}$ analysis of the distribution of chemotherapy cycles with different platinum agent groups in the subgroups. DDP, cisplatin; NDP, nedaplatin. 
Table III. Log-rank test for comparing overall survival time in the subgroups.

\begin{tabular}{|c|c|c|c|c|c|}
\hline \multirow[b]{2}{*}{ Variables } & \multirow[b]{2}{*}{$\mathrm{n}$} & \multicolumn{2}{|c|}{ Median survival time $(95 \% \mathrm{CI})$} & \multirow[b]{2}{*}{$\chi^{2}$} & \multirow[b]{2}{*}{ P-value } \\
\hline & & $\operatorname{DDP}(n=202)$ & $\operatorname{NDP}(n=190)$ & & \\
\hline \multicolumn{6}{|l|}{ Gender } \\
\hline Female & 111 & $16(15.1-16.9)$ & $16(9.9-22.1)$ & 0.582 & 0.445 \\
\hline Male & 281 & $14(11.7-16.3)$ & $20(16.8-23.2)$ & 14.225 & $<0.001$ \\
\hline \multicolumn{6}{|l|}{ Age, years } \\
\hline$<60$ & 212 & $16(14.5-17.5)$ & $21(12.4-29.6)$ & 21.121 & $<0.001$ \\
\hline$\geq 60$ & 180 & $14(11.6-17.3)$ & $16(13.2-17.8)$ & 0.068 & 0.795 \\
\hline \multicolumn{6}{|l|}{ Smoking status } \\
\hline Non-smoker & 194 & $16(14.4-17.6)$ & $20(14.6-25.4)$ & 7.029 & 0.008 \\
\hline Current smoker & 198 & $15(10.2-19.8)$ & $19(16.2-21.2)$ & 6.217 & 0.013 \\
\hline \multicolumn{6}{|l|}{ Histology type } \\
\hline $\mathrm{Sq}$ & 132 & $16(10.4-19.6)$ & $24(20.4-31.6)$ & 10.305 & $<0.001$ \\
\hline Non-Sq & 260 & $15(14.4-17.6)$ & $17(14.8-21.2)$ & 0.345 & 0.557 \\
\hline \multicolumn{6}{|l|}{ Stage } \\
\hline I-II & 40 & $34(20.6-40.4)$ & $38(30.1-45.9)$ & 0.669 & 0.413 \\
\hline III & 209 & $15(12.7-17.3)$ & $20(18.1-21.9)$ & 5.360 & 0.021 \\
\hline IV & 143 & $10(9.0-11.0)$ & $14(12.5-15.5)$ & 2.508 & 0.113 \\
\hline \multicolumn{6}{|l|}{ Cycles } \\
\hline $2-3$ & 184 & $10(7.8-12.2)$ & $12(10.3-13.7)$ & 5.106 & 0.204 \\
\hline $4-5$ & 148 & $18(14.5-22.5)$ & $20(15.9-28.1)$ & 0.053 & 0.818 \\
\hline$\geq 6$ & 60 & $22(20.9-30.1)$ & $24(14.0-36.0)$ & 1.121 & 0.290 \\
\hline
\end{tabular}

CI, confidence interval; Sq, squamous cancer; GP, gemcitabine + platinum; TP, paclitaxel + platinum; DP, docetaxel + platinum; CAP, cyclophosphamide + doxorubicin + platinum; NP, navelbine + platinum.

Table IV. Toxicity of grades 3-4 of different platinum agents.

\begin{tabular}{lcccc}
\hline Variables & DDP, n (\%) & NDP, n (\%) & $\chi^{2}$ & P-value \\
\hline Hematologic & & & & \\
Anemia & $8(4.0)$ & $3(1.6)$ & 2.036 & 0.154 \\
Neutropenia & $42(20.8)$ & $32(16.8)$ & 0.998 & 0.192 \\
Thrombocytopenia & $11(5.4)$ & $23(12.1)$ & 5.482 & 0.019 \\
Non-hematologic & & & & \\
Nausea/vomiting & $73(36.1)$ & $16(8.4)$ & 48.862 & $<0.001$ \\
Anorexia & $35(17.3)$ & $11(5.8)$ & 12.582 & $<0.001$ \\
Renal toxicity & $0(0.0)$ & $0(0.0)$ & N.A. & N.A. \\
Neurotoxicity & $0(0.0)$ & $0(0.0)$ & N.A. & N.A. \\
Weight loss & $20(9.9)$ & $2(1.0)$ & 13.642 & $<0.001$ \\
\hline
\end{tabular}

DDP, cisplatin; NDP, nedaplatin; N.A., not accessible.

with NSCLC. For a number of years, DDP has been the major agent in these regimens. However, its relatively high rates of renal and gastrointestinal toxicities lead numerous patients in China to give up chemotherapy. Carboplatin and NDP are DDP analogs, with a relatively lower toxicity profile. As NDP has the same administration method and dosage as DDP, it has become the most popular platinum-based agent for NSCLC patients in China. Although numerous trials have compared the effect and survival benefit of DDP and carboplatin in NSCLC (18-21), only few trials have compared NDP with DDP. Cao et al (22) reported that NDP had similar response rates to DDP in the treatment of nasopharyngeal carcinoma. Yamashita et al (23) reported that the overall survival rates of NDP at 1, 2 and 3 years were lower than those of DDP (40, 13 and $13 \%$ vs. 56,42 and $8 \%$, respectively) in the treatment of esophageal cancer, but no significant difference was found between the two groups. However, in the study, the two groups had an unequal number of patients (12 on the NDP regimen vs. 29 on the DDP regimen). Therefore, the survival benefit of NDP has remained an unsolved issue thus far.

In the present study, the patients receiving NDP-based chemotherapy had higher survival rates than those treated with DDP. The MST was greater by 5 months, whereas the 1and 2-year overall survival rates were also higher in the NDP group. The observed survival benefits of NDP can be explained as follows: Firstly, the two groups have similar baseline characteristics, except for the chemotherapy cycles. As patients receiving NDP-based treatment experience less toxicity and show good compliance with the chemotherapy regimen, these patients can complete more cycles of the chemotherapy. The chemotherapy cycle number was an independent predictive factor. More chemotherapy cycles can reduce the mortality risk for $46 \%$. Scotti et al (24) also came to the same conclusion 
that the number of chemotherapy courses persisted as a significant mortality predictor at multivariate regression analysis, with a reduced mortality risk for 5-6 chemotherapy cycles in comparison to 3-4 cycles (HR, 0.44). Secondly, the differential weight loss effect of NDP could also account for the observed survival benefit. More specifically, the rate of weight loss was much higher in DDP- compared to NDP-treated patients. Yang et al (25) reported that lung cancer patients undergoing weight loss had shorter MST than those not losing weight (6.4 vs. 9.2 months, $\mathrm{P}<0.001)$. Finally, the present study also showed that the type of platinum agent used was an independent predictive factor for the overall survival time and HR is 0.764. Preclinical and in vitro studies have found that the plasma concentration profile of unbound platinum following NDP infusion is similar to that of total platinum, and that the protein-binding affinity of NDP is lower than that of DDP (26). Thus, NDP has been demonstrated to have higher antitumor activity than DDP (2).

In the present study, male patients, patients $<60$ years of age, and patients with squamous cancer and stage III in the NDP group had a much longer survival time than patients with similar characteristics in the DDP group. In the further study, the distribution of chemotherapy cycles was significantly different in different platinum agent groups, which suggested that the chemotherapy cycles were the main reason that caused the different survival time. However, Yamamoto et al (27) observed that when NDP was used in advanced NSCLC patients, partial responses were observed in $13(33 \%)$ of the 39 patients, while 12 of the 13 patients who responded had squamous cell carcinoma. Teramoto et al (28) also reported that NDP responded better in squamous cell carcinoma of the lung. Thus, further clinical trials are required to confirm these observations.

In conclusion, NDP-based chemotherapy prolongs the median survival time of NSCLC patients, compared to DDP-based chemotherapy. The observed survival benefit is due to the reduced toxicity of NDP, which allows patients to tolerate more cycles of chemotherapy. A slow toxicity and high life quality were the tendencies of the advanced cancer treatment currently. Thus, NDP may be a more reasonable choice than DDP in clinical practice. In addition, noteworthy information is also provided regarding the impact of gender, age and histological type, which may improve treatments by targeting specific patient populations.

\section{Acknowledgements}

The authors thank the Chinese National Natural Science Foundation (grant nos. 30801367 and 81272599). They also thank the colleagues of the Cancer Centre (Daping Hospital) for collecting the patient information, including Mrs. Lei Xia, Mrs. Juan Li, Mrs. Wei Luo, Mrs. Shuai Wang, Mrs. Yan Feng, Mrs. Qian Zhou, Mrs. Hong Peng and Miss Shu Chen. Thank you to Editage for providing editorial and publication support.

\section{References}

1. Pastorino U: Lung cancer screening. Br J Cancer 102: 1681-1686, 2010.

2. Kanzawa F, Matsushima Y, Nakano H, et al: Antitumor activity of a new platinum compound (glycolate-o,o') diammineplatinum (II) (254-S), against non-small cell lung carcinoma grown in a human tumor clonogenic assay system. Anticancer Res 8: 323-327, 1998.
3. Suzumura Y, Kato T, Ueda R and Ota K: Effect of treatment schedule on antitumor activity of glycolate- 0,0 '-diammineplati num(II), a new platinum derivative: comparison with cis-diam minedichloroplatinum(II). Anticancer Res 9: 1083-1088, 1989.

4. Hida S, Okada K and Yoshida O: Advantages in combination chemotherapy using cisplatin and its analogues for human testicular tumor xenografts. Jpn J Cancer Res 81: 425-430, 1990.

5. Koshiyama M, Kinezaki M, Uchida T and Sumitomo M: Chemosensitivity testing of a novel platinum analog, nedaplatin (254-S), in human gynecological carcinomas: a comparison with cisplatin. Anticancer Res 25: 4499-4502, 2005.

6. Kuruse K, Fukuoka M, et al: A phase II clinical study of cis-diammine glycolato platinum, 254-S, for primary lung cancer. Gan To Kagaku Ryoho 19: 879-884, 1992.

7. Zheng J, Wang G, Yang GY, et al: Induction chemotherapy with nedaplatin with 5-FU followed by intensity-modulated radiotherapy concurrent with chemotherapy for locoregionally advanced nasopharyngeal carcinoma. Jpn J Clin Oncol 40: 425-431, 2010.

8. Naito Y, Kubota K, Ohmatsu H, et al: Phase II study of nedaplatin and docetaxel in patients with advanced squamous cell carcinoma of the lung. Ann Oncol 22: 2471-2475, 2011.

9. Oshita F, Ohe M, Honda T, et al: Phase II study of nedaplatin and irinotecan with concurrent thoracic radiotherapy in patients with locally advanced non-small-cell lung cancer. Br J Cancer 103: 1325-1330, 2010.

10. Osawa S, Furuta T, Sugimoto K, et al: Prospective study of daily low-dose nedaplatin and continuous 5-fluorouracil infusion combined with radiation for the treatment of esophageal squamous cell carcinoma. BMC Cancer 9: 408, 2009.

11. Jin J, Xu X, Wang F, et al: Second-line combination chemotherapy with docetaxel and nedaplatin for Cisplatin-pretreated refractory metastatic/recurrent esophageal squamous cell carcinoma. J Thorac Oncol 4: 1017-1021, 2009.

12. Kitamura H, Taguchi K, Kunishima Y, et al: Paclitaxel, ifosfamide and nedaplatin as second-line treatment for patients with metastatic urothelial carcinoma: a phase II study of the SUOC group. Cancer Sci 102: 1171-1175, 2011.

13. Yin M, Zhang H, Li H, et al: The toxicity and long-term efficacy of nedaplatin and paclitaxel treatment as neoadjuvant chemotherapy for locally advanced cervical cancer. J Surg Oncol 105: 206-211, 2012.

14. Sasaki Y, Saijo N and Tamura T: Comparison of the antitumor activity of cisplatin and its derivatives with special stress on the pharmacokinetics of active form of drugs in the plasma determined by colony assay. Proc Am Soc Clin Oncol 6: 34, 1987.

15. Furuse K, Fukuoka M, Asamoto H, et al: A randomized comparative study of 254-S plus vindesine (VDS) vs. cisplatin (CDDP) plus VDS in patients with advanced non-small cell lung cancer (NSCLC). Gan To Kagaku Ryoho 19: 1019-1026, 1992.

16. Guo JF, Zhang B, Wu F, et al: A phase II trial of docetaxel plus nedaplatin and 5-fluorouracil in treating advanced esophageal carcinoma. Chin J Cancer 29: 348-352, 2010.

17. Cancer Therapy Evaluation Program, Common Terminology Criteria for Adverse Events, Version 3.0, DCTD, NCI, NIH, DHHS, 2006

18. Ohe Y, Ohashi Y, Kubota K, et al: Randomized phase III study of cisplatin plus irinotecan versus carboplatin plus paclitaxel, cisplatin plus gemcitabine and cisplatin plus vinorelbine for advanced non-small-cell lung cancer: four-arm cooperative study in Japan. Ann Oncol 18: 317-323, 2007.

19. Hotta K, Matsuo K, Ueoka H, Kiura K, Tabata M and Tanimoto M: Meta-analysis of randomized clinical trials comparing Cisplatin to Carboplatin in patients with advanced non-small-cell lung cancer. J Clin Oncol 22: 3852-3859, 2004.

20. Jiang J, Liang X, Zhou X, Huang R and Chu Z: A meta-analysis of randomized controlled trials comparing carboplatin-based to cisplatin-based chemotherapy in advanced non-small cell lung cancer. Lung Cancer 57: 348-358, 2007.

21. Ardizzoni A, Boni L, Tiseo M, et al: Cisplatin- versus carboplatin-based chemotherapy in first-line treatment of advanced non-small-cell lung cancer: an individual patient data meta-analysis. J Natl Cancer Inst 99: 847-857, 2007.

22. Cao KJ, Zhang AL, Ma WJ, Huang PY, Luo DH and Xia WX: Nedaplatin or cisplatin combined with 5-fluorouracil for treatment of stage III-IVa nasopharyngeal carcinoma: a randomized controlled study. Zhonghua Zhong Liu Za Zhi 33: 50-52, 2011. 
23. Yamashita H, Nakagawa K, Tago M, et al: Radiation therapy combined with cis-diammine-glycolatoplatinum (nedaplatin) and 5-fluorouracil for Japanese stage II-IV esophageal cancer compared with cisplatin plus 5-fluorouracil regimen: a retrospective study. Dis Esophagus 19: 15-19, 2006.

24. Scotti V, Meattini I, Saieva C, et al: Limited-stage small-cell lung cancer treated with early chemo-radiotherapy: the impact of effective chemotherapy. Tumori 98: 53-59, 2012.

25. Yang R, Cheung MC, Pedroso FE, Byrne MM, Koniaris LG and Zimmers TA: Obesity and weight loss at presentation of lung cancer are associated with opposite effects on survival. J Surg Res 170: e75-e83, 2011.
26. Ota K, Oguma T and Shimamura K: Pharmacokinetics of platinum in cancer patients following intravenous infusion of cis-diammine (glycolato) platinum, 254-S. Anticancer Res 14: 1383-1387, 1994

27. Yamamoto N, Tamura T, Kurata T, et al: Dose-finding and pharmacokinetic study of nedaplatin in elderly patients with advanced non-small cell lung cancer. Cancer Chemother Pharmacol 65: 79-88, 2009.

28. Teramoto K, Asada Y, Ozaki Y, et al: phase II study of docetaxel plus nedaplatin in patients with metastatic non-small-cell lung cancer. Cancer Chemother Pharmacol 70: 531-537, 2012. 